\title{
Kommentar zum Sustainable Supply Chain Management
}

\author{
Anmerkungen zum Beitrag \\ Hansen, Erik G./Harms, Dorli/Schaltegger, Stefan: Sustainable Supply Chain \\ Management im globalen Kontext - Praxisstand des Lieferantenmanagements in \\ DAX- und MDAX-Unternehmen
}

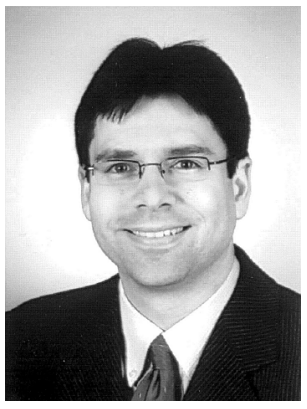

\section{Frank Teuteberg}

Seit Mitte der 80er Jahre werden in Wissenschaft und Unternehmenspraxis unterschiedliche Konzepte im Supply Chain Management (SCM) diskutiert. Den traditionellen SCM-Konzepten stehen jedoch vermehrt neue Herausforderungen gegenüber, wie bspw. eine Vielzahl von (neuen) regulatorischen und gesetzlichen Anforderungen zum Umweltschutz (z. B. WEEE, ElektroG, ElektroGKostV, BattV, PCB AbfallV), Normen und Standards (z. B. Energy Star Computer Program, TCO), erweiterte Berichts- und Publizitätspflichten zum nachhaltigen Wirtschaften (Sustainability Index, EMAS) sowie die Verknappung der natürlichen Ressourcen. Hinzu kommen ein wachsendes Interesse der Öffentlichkeit am Umweltschutz (Green Logistics) und die Forderung nach einem ausreichenden Schutz von Mitarbeitern, die mit giftigen Gefahrenstoffen (z. B. PVC, bromierten Flammschutzmitteln, Chlorgehalt auf Leiterplatten) in Kontakt kommen. Ebenso wird von den Kunden der Unternehmen vermehrt ein verantwortungsvollerer Umgang mit Mitarbeitern (Social and Ethical Auditing, Accounting and Reporting (SEAAR), Ethical Trading Initiative and Supplier Ethical Data Exchange (ETI)) eingefordert. Auch aufgrund der zunehmenden Komplexität von globalen Lieferanten-Abnehmer-Beziehungen ist es unabdingbar, geeignete Methoden, Informations- und Kommunikationssysteme (IuK-Systeme) sowie Technologien für ein umweltorientiertes, soziales und langfristig profitables Lieferantenmanagement einzusetzen. Das Sustainable Supply Chain Management (SSCM) erweitert die Konzepte des SCM um umweltorientierte und soziale Aspekte und trägt damit den Forderungen nach mehr Nachhaltigkeit Rechnung (Seuring/Müller 2007).

Erik G. Hansen, Dorli Harms und Stefan Schaltegger gehen in ihrem Beitrag "Sustainable Supply Chain Management im globalen Kontext - Praxisstand des Lieferantenmanagements in DAX- und MDAX-Unternehmen " der Frage nach, wie das SSCM in der Unternehmenspraxis derzeit umgesetzt wird und welche Massnahmen von DAX- und MDAX-Unternehmen, insbesondere im Bereich des Lieferantenmanagements, getroffen werden. Ihr komparativer Vergleich zeigt auf, dass DAX-Unternehmen in der Ausgestaltung des SSCM weiter als MDAX-Unternehmen sind und Umsetzungsmassnahmen des SSCM sowohl häufiger als auch standardisierter anwenden. Ressourcenaufwendigere Bewertungs- und Audit-Verfahren zur nachhaltigen Lieferantenentwicklung sind jedoch im Vergleich zu einfachen Umsetzungsmassnahmen (z. B. schriftliches Erfassen von Anforderungen) insgesamt unterrepräsentiert. Die steigende Bedeutung eines nachhaltigen Liefe- 
rantenmanagements wird jedoch sowohl bei den DAX- als auch bei den MDAX-Unternehmen deutlich.

Dennoch stellt sich dem Leser der Studie von Erik G. Hansen, Dorli Harms und Stefan Schaltegger die Frage, weshalb noch nicht alle Unternehmen konsequent umfassende Massnahmen für ein nachhaltiges Lieferantenmanagement ergreifen. Fehlt es an dem entsprechenden Nachhaltigkeits-Bewusstsein? Fehlt es an Software-Unterstützung zur Performance-Messung einer nachhaltigen Lieferantenentwicklung? Sind die Zusammenhänge zwischen ökologischen, ökonomischen und sozialen Massnahmen zu komplex? Fehlt es an Anreizsystemen für die Verantwortlichen in Massnahmen für ein nachhaltiges Lieferantenmanagement zu investieren? Stehen andere Themen derzeit noch höher auf der Agenda der Unternehmen? Welche Anreize haben Verantwortliche und Arbeitnehmer überhaupt, energie- und ressourceneffizient zu handeln und ihre Lieferanten unter Nachhaltigkeitsaspekten auszuwählen, wo doch ihr persönliches Gehalt in der Regel nicht an die Energiekosten oder Ressourcenverbräuche des Unternehmens gekoppelt ist?

Nach Auffassung des Autors dieser Replik bedarf es eines strategischen Umdenkens und weiterführender Massnahmen als diejenigen, die allgemein in der Literatur unter dem Begriff Lieferantenmanagement subsummiert werden. In der einschlägigen Literatur zur Lieferantenauswahl werden überwiegend die Kriterien Kosten, Zeit und Qualität diskutiert (Sarkis 1998). Darüber hinaus berücksichtigen einige wenige Autoren umweltorientierte Kriterien für die Auswahl von Lieferanten (Sarkis1998; Sarmiento/Thomas 2010; Su et al. 2010). Eine ganzheitliche und integrierte Betrachtung von umweltorientierten, sozialen und ökonomischen Kriterien findet sich dagegen in der Literatur nur vereinzelt.

Häufig wird in der öffentlichen und mitunter auch in der wissenschaftlichen Diskussion der Begriff der Nachhaltigkeit zudem weitgehend sinnentleert verwendet oder zu Marketingzwecken in der Unternehmenspraxis missbraucht (sog. „Greenwashing“). Das Thema „Nachhaltigkeit“ ist jedoch ein sehr vielschichtiges und komplexes Themengebiet wie der Beitrag von Erik G. Hansen, Dorli Harms und Stefan Schaltegger aufzeigt. Die strategische Komponente eines Sustainable Supply Chain Managements ist (noch) nicht bei allen Unternehmen angekommen, wie auch zahlreiche Industrie-Workshops und Studien (Wittstruck/Teuteberg 2011 a) des Autors dieser Replik ergeben haben. Die umwelt- und soziale Komponente der Nachhaltigkeit ist ebenfalls (noch) nicht umfassend in das Zielsystem der Unternehmen integriert.

Nachbaltiges Lieferantenmanagement sollte jedoch bereits zu Beginn der Entwicklung von (hybriden) Produkten, im Rahmen einer umweltintegrierten Produktion sowie in der strategischen Entscheidungsfindung und nicht erst als sog. „End-of-Pipe“-Lösung zur Dokumentation von Umweltkennzahlen und zur Herstellung von Legal Compliance stattfinden.

Durch den Einsatz intelligent vernetzter Systeme und Prozesse könnten Informationsund Kommunikationssysteme (IuK) helfen, bspw. auf der strategischen Unternehmensebene strategisch relevante Umweltinformationen und Entscheidungsalgorithmen zur Verfügung zu stellen, die zu einer besseren Einschätzung nachhaltiger Entwicklungspfade, erfolgskritischer Ressourcenpreise bzw. volatiler Energiemärkte führen und damit einhergehende Risiken sowie strategisch relevante, systemdynamische Ursache-Wirkungszusammenhänge zwischen ökonomischen, ökologischen und sozialen Indikatoren sichtbar machen (Wittstruck/Teuteberg 2011 b). 
Erik G. Hansen, Dorli Harms und Stefan Schaltegger ist beizupflichten, dass es an (empirischen) Untersuchungen mangelt, welche den konkreten Beitrag eines nachhaltigen Lieferantenmanagements/SSCM zum ökologischen, ökonomischen und sozialen Erfolg (Umweltleistung bzw. Nachhaltigkeit) von Unternehmen untersuchen. Die folgende Frage bleibt demnach auch für viele Unternehmen unbeantwortet: Welche Einflussfaktoren, Unternehmenskulturen sowie welche Massnahmen (organisatorisch sowie Investitionen in ITArchitekturen) haben welchen konkreten Einfluss auf die Umweltleistung (z. B. Recyclingquote, Energie- und Ressourcenverbräuche bzw. Energieeffizienz, $\mathrm{CO}^{2}$-Footprint, etc.) in der Unternehmenspraxis?

Wittstruck/Teuteberg (2011 a) haben auf der Basis einer empirischen Studie ein Erklärungsmodell entwickelt, welches Faktoren aufzeigt, die den Erfolg des Sustainable Supply Chain Management in Unternehmen beeinflussen. Das Konstrukt „Erfolg“ wird von den Autoren dabei anhand der Grössen „Unternehmensimage“, „Prozesstransparenz“, „Performance“ sowie „Energie- und Ressourceneffizienz“ gemessen. Wesentliche Faktoren für ein erfolgreiches Sustainable Supply Chain Management, so zeigt die Befragung von 115 Unternehmen, sind „Signaling“, Informationsbereitstellung und -austausch auf der Basis von IuK-Systemen, die Einführung und Abstützung auf Standards (z. B. Green SCOR), das strategische Bekenntnis der Netzwerkpartner zum SSCM, ein kontinuierliches Lernen sowie die Berücksichtigung ökologischer Kreisläufe bei der Entwicklung und dem Recycling von Produkten.

Aus Sicht des Autors dieser Replik fehlt es in der Unternehmenspraxis insbesondere an Informationssystemen, die sich in einer ganzheitlichen Weise mit Material- und Energieeffizienz, Emissions- und Abfallreduktion, Entsorgung, der Erfüllung gesetzlicher Vorschriften und dem strategischem Lieferantenmanagement auseinandersetzen. Derartige IuK-Systeme existieren in der wissenschaftlichen Diskussion bisher nur als Konzepte. Um sie zum Einsatz zu bringen, bedarf es intensiver Forschung sowie verstärkter Transferarbeit bei KMUs. Derartige Systeme könnten Unternehmen in die Lage versetzen

- umweltfreundlichere Produktions- und Entsorgungsverfahren zu entwickeln,

- hybride Produkte zu entwickeln, bei denen der Nachhaltigkeitsgedanke stärker im Vordergrund steht (diese hybriden Produkte integrieren sowohl Sach- als auch Dienstleistungen und werden als Leistungsbündel am Markt angeboten),

- den interaktiven Informationsaustausch mit verschiedenen Stakeholdern/Lieferanten im Bereich der Nachhaltigkeitsberichterstattung auf der Basis von Web 2.0 Technologien (Blogs, Wiki, Semantic Web, Podcasts, etc.) zu realisieren,

- Synergieeffekte, Kosteneinsparungen, strategische Vorteile etc. durch Angebot und Nutzung von „grünen“ Diensten aus der Cloud („Sustainable Cloud Computing“) und auf der Basis von Auktionsplattformen zu realisieren,

- aus strategischer Sicht komplementäre Ursache-Wirkungsbeziehungen zwischen ökonomischen, sozialen und ökologischen Zielen aufzuzeigen. Komplementär bedeutet in diesem Zusammenhang, dass die Verfolgung eines (bspw. ökologischen) Ziels das Erreichen anderer (wie sozialer oder ökonomischer) Ziele unterstützt.

Bestehende Methoden, Konzepte und Referenzmodelle wie die Sustainable Balanced Scorecard, Potentialmodellierung, Self-Assessments auf der Basis von Checklisten und Reifegradmodellen sowie Referenzmodelle (Best Practices) wie ITIL, Cobit, Eco-Integral, GreenSCOR können eine erste Grundlage für die Entwicklung strategischer Konzepte im Rahmen des SSCM sowie für die Etablierung eines nachhaltigen Lieferantenmanagements 
bieten. Sie können - zumindest ansatzweise - die oben beklagte (strategische) Lücke und das Fehlen ganzheitlicher, integrierter IuK-Systeme zur Unterstützung eines nachhaltigen Lieferantenmanagements schliessen. Zu einer gehaltvollen Anwendung der vorhandenen Methoden, Konzepte, IuK-Systeme und Referenzmodelle ist jedoch die Entwicklung und Etablierung von (langfristig orientierten) Zielsystemen der Nachhaltigkeit in den Unternehmen erforderlich.

\section{Literaturhinweise}

Lu, L.Y.Y., et al. (2007): Environmental principles applicable to green supplier evaluation by using multi-objective decision analysis, in: International Journal of Production Research, Jg. 45, Nr. 18-19, S. 4317-4331.

Sarkis, J. (1998): Evaluating environmentally conscious business practices, in: European Journal of Operational Research, Jg. 107, Nr. 1, S. 159-174.

Sarmiento, R./Thomas, A. (2010): Identifying improvement areas when implementing green initiatives using a multitier AHP approach, in: Benchmarking: An International Journal, Jg. 17, Nr. 3, S. 452-463.

Seuring, S./Müller, M. (2007): Core issues in sustainable supply chain management - a Delphi study, in: Business Strategy and the Environment, Jg. 17, Nr. 8, S. 455-466.

Su, Y., et al. (2010): System Dynamics Modeling for Strategic Management of Green Supply Chain, in: Strategic Information Systems: Concepts, Methodologies, Tools, and Applications, Hunter, M.G., S. 2617-2649.

Wittstruck, D./Teuteberg, F. (2011a): Understanding the Success Factors of Sustainable Supply Chain Management: Empirical Evidence from the Electrics and Electronics Industry, in: Corporate Social Responsibility and Environmental Management (vormals Eco-Management and Auditing), (Angenommen zur Publikation: Februar 2011).

Wittstruck, D./Teuteberg, F. (2011 b): Development and Simulation of a Balanced Scorecard for Sustainable Supply Chain Management - A System Dynamics Approach, in: Proceedings der 10. Internationalen Tagung Wirtschaftsinformatik, Zürich 2011 (nominiert für den OUTSTANDING PAPER AWARD).

Frank Teuteberg, Prof. Dr., ist Fachgebietsleiter für Unternehmensrechnung und Wirtschaftsinformatik am Institut für Informationsmanagement und Unternehmensführung der Universität Osnabrück.

Anschrift: Universität Osnabrück, Fachgebiet Unternehmensrechnung und Wirtschaftsinformatik, Katharinenstr. 1, D-49069 Osnabrück, Tel.: +49 (0)541/969-4961, Fax: +49 (0)541/969-14961, E-Mail: frank.teuteberg@uni-osnabrueck.de 